STUPP-95-139

May, 1995

\title{
General considerations of matter coupling with the self-dual connection
}

\author{
Motomu Tsuda, Takeshi Shirafuji and Hong-Jun Xie \\ Department of Physics, Saitama University \\ Urawa, Saitama 338, Japan
}

\begin{abstract}
It has been shown for low-spin fields that the use of only the self-dual part of the connection as basic variable does not lead to extra conditions or inconsistencies. We study whether this is true for more general chiral action. We generalize the chiral gravitational action, and assume that half-integer spin fields are coupled with torsion linearly. The equation for torsion is solved and substituted back into the generalized chiral action, giving four-fermion contact terms. If these contact terms are complex, the imaginary part will give rise to extra conditions for the gravitational and matter field equations. We study the four-fermion contact terms taking spin-1/2 and spin-3/2 fields as examples.
\end{abstract}




\section{Introduction}

In the mid-1980s Ashtekar has presented a new formulation of general relativity from a non-perturbative point of view, in terms of which all the constraints of the gravity become simple polynomials of complex canonical variables [1]- [4]. It is possible to arrive at this formulation from a first-order Palatini-type action in which a tetrad and a complex self-dual connection are regarded as independent variables [4. Moreover, this formulation can be extended to include matter sources. In particular, it has been shown that the constraints are again polynomials of the canonical variables in the cases of spin-1/2 fields [4, 5] and $N=1$ supergravity [3, 6, 7]. The recent progress can be traced from the references compiled in [8].

It is the purpose of this paper to discuss the consistency of the field equations in the Ashtekar formalism. For definiteness, we shall restrict ourselves to the self-dual case throughout this paper. The chiral action $S^{(+)}$using the self-dual connection can be expressed as «1]

$$
\begin{aligned}
S^{(+)}= & {[\text {Einstein-Hilbert action }] } \\
& +[\text { quadratic terms of torsion }]+[\text { matter terms }] .
\end{aligned}
$$

The total divergence terms of the action, if they arise, are omitted throughout this paper, because they do not contribute to the field equations. Since the torsion vanishes in the source-free case, the complex chiral action is equivalent to the Einstein-Hilbert action. If the matter terms (in particular, half-integer spin fields) exist, however, the imaginary part will emerge from the quadratic terms of torsion and from the matter terms in (1.1). If this is indeed the case, the field equations will be affected by the imaginary part of the chiral action.

In 1973 Hayashi and Bregman generalized the gravitational Lagrangian of the Poincaré gauge theory by adding the most general quadratic terms of torsion to the first-order Palatini Lagrangian [9]. It is easy to set up the chiral version of their generalization. When matter fields of half-integer spin exist, the quadratic terms of torsion in the generalized chiral action differ from those of (1.1). So it is of interest to study how the gravitational and matter field equations are affected by such a generalization.

As for the matter coupling, we shall obtain the matter Lagrangian by the minimal

prescription which employs only the self-dual connection. The Lagrangian of Dirac 
fields and Rarita-Schwinger fields is, as is well-known, linear in first derivatives of the field variables, and the torsion is linearly coupled with these fields. Therefore, we assume that the matter Lagrangian for half-integer spin fields involves the first order derivatives and hence the torsion linearly.

We are thus led to the generalized chiral action which is schematically expressed as follows:

$$
\begin{aligned}
S^{(+)}= & {[\text {Einstein-Hilbert action }]+S_{M}(e) } \\
& +[\text { general quadratic terms of torsion }] \\
& +[(\text { torsion }) \times(\text { matter fields })]
\end{aligned}
$$

where $S_{M}(e)$ is the matter action in general relativity. The equation of motion for the torsion can be derived from (1.2), and can easily be solved with respect to the torsion. Substituting the solution back into the torsion part of (1.2) gives the fourfermion contact terms of the generalized chiral action. If these contact terms are complex, there is a possibility that consistency problem arises in the gravitational and matter field equations.

It has been shown for spin-1/2 fields [4, 5] and simple supergravity [3, 6, 7] that the imaginary part of the chiral action vanishes. We investigate in this paper whether the imaginary part of the four-fermion contact terms vanishes or not for generic half-integer spin fields which is assumed to couple with the torsion linearly.

For convenience of calculation, a tensor quantity is introduced, which is denoted by $L_{i j \mu}$ and related to the torsion, by taking the covariant differentiation of the quadratic form of the tetrad field and by totally anti-symmetrizing three space-time indices. I This quantity will enable us to solve the equation of motion for the self-dual part of the contorsion tensor in a simple and transparent manner.

The present paper is organized as follows. In Sec.2, following Hayashi and Bregman, we generalize the chiral gravitational Lagrangian. In Sec.3 we discuss the half-integer spin fields minimally coupled to gravity. Based on the considerations of spin-1/2 and spin-3/2 fields, the general form of the chiral Lagrangian of matter fields is set up assuming that the torsion is coupled with the matter fields linearly. In Sec. 4 we derive the equation of motion for the torsion from the generalized chiral

\footnotetext{
${ }^{*}$ Greek letters $\mu, \nu, \cdots$ are space-time indices, and Latin letters $i, j, \cdots$ are local Lorentz indices. We denote the Minkowski metric by $\eta_{i j}=\operatorname{diag}(-1,+1,+1,+1)$. The totally antisymmetric tensor $\epsilon_{i j k l}$ is normalized as $\epsilon_{0123}=+1$.
} 
Lagrangian, and solve it with respect to the torsion. In Sec.5 the consistency of the field equations is analyzed. Spin-1/2 fields and (Majorana) Rarita-Schwinger fields are considered as examples. In the final section our results are summarized.

\section{Chiral gravitational Lagrangian and its generalization}

We consider space-time manifold with a metric field $g_{\mu \nu}$ constructed from a tetrad field $e_{\mu}^{i}$ via $g_{\mu \nu}=e_{\mu}^{i} e_{\nu}^{j} \eta_{i j}$, and denote a Lorentz connection by $A_{i j \mu}=A_{[i j] \mu}$. ⺆ In the first-order Palatini framework, independent dynamical variables are the tetrad and the Lorentz connection. On the other hand, the tetrad and the complex self-dual connection are used as the dynamical variables in the chiral action. The self-dual connection $A_{i j \mu}^{(+)}$is defined by

$$
A_{i j \mu}^{(+)}:=\frac{1}{2}\left(A_{i j \mu}-\frac{i}{2} \epsilon_{i j}{ }^{k l} A_{k l \mu}\right)
$$

The complex chiral action using the tetrad and the self-dual connection is

$$
S^{(+)}=\int d^{4} x e R^{(+)}+[\text {matter terms }]
$$

where $e=\operatorname{det}\left(e_{\mu}^{i}\right)$ and

$$
R^{(+)}=\frac{1}{2}\left(R_{\mu \nu}^{i j}-\frac{i}{2} \epsilon_{k l}^{i j} R_{\mu \nu}^{k l}\right) e_{i}^{\mu} e_{j}^{\nu}
$$

Here $R^{i j}{ }_{\mu \nu}$ is the curvature tensor written in terms of the Lorentz connection, and we use unit with $8 \pi G=c=1$. The $R^{(+)}$of $(2.3)$ is also given by the self-dual connection as

$$
R^{(+)}=\left(\partial_{\mu} A_{\nu}^{(+) i j}-\partial_{\nu} A_{\mu}^{(+) i j}+A_{k \mu}^{(+) i} A_{\nu}^{(+) k j}-A_{k \nu}^{(+) i} A_{\mu}^{(+) k j}\right) e_{i}^{\mu} e_{j}^{\nu} .
$$

\footnotetext{
* The antisymmetrization of a tensor is denoted by $A_{[i j]}:=(1 / 2)\left(A_{i j}-A_{j i}\right)$.
} 
In order to separate the quadratic terms of torsion from the gravitational part of the chiral action in a transparent manner, we introduce the following quantity:

$$
L^{i j \mu}:=-\frac{1}{4} \epsilon^{i j}{ }_{m n} \epsilon^{\mu \nu \rho \sigma} D_{\nu} H_{\rho \sigma}^{m n},
$$

where $H^{i j}{ }_{\mu \nu}$ is defined by

$$
H_{\mu \nu}^{i j}:=2 e_{[\mu}^{i} e_{\nu]}^{j}
$$

and the covariant derivative $D_{\mu}$ acts only on local Lorentz indices giving

$$
D_{\mu} e_{\nu}^{i}=\partial_{\mu} e_{\nu}^{i}+A_{j \mu}^{i} e_{\nu}^{j}
$$

The Lorentz connection is divided into the Ricci rotation coefficients $A_{i j \mu}(e)$ and contorsion tensor $K_{i j \mu}$ :

$$
A_{i j \mu}=A_{i j \mu}(e)+K_{i j \mu}
$$

Using (2.8) in (2.7) gives

$$
D_{[\mu} e_{\nu]}^{i}=K_{j[\mu}^{i} e_{\nu]}^{j}=-\frac{1}{2} T_{\mu \nu}^{i}
$$

with $T_{\mu \nu}^{i}$ being the torsion tensor. Then the $L^{i j \mu}$ of (2.5) can be rewritten as

$$
L^{i j \mu}=-\frac{1}{2} \epsilon_{m n}^{i j} \epsilon^{\mu \nu \rho \sigma} e_{\nu}^{m} T_{\rho \sigma}^{n}
$$

which can be solved with respect to $T_{\mu i j}$ as follows:

$$
T_{\mu i j}=-L_{i j \mu}-e_{\mu[i} L_{j] k}^{k}
$$

Furthermore, $L_{i j \mu}$ can also be expressed by the contorsion tensor as

$$
L_{i j \mu}=2\left(e_{\mu[i} K_{j] k}^{k}-K_{\mu[i j]}\right) .
$$

With the help of this relation (2.12), the scalar curvature can be written as

$$
R=R(e)+\frac{1}{2} L_{i j \mu} K^{i j \mu}
$$


where $R(e)$ is the Riemann-Christoffel scalar curvature.

The self-dual part of $L^{i j \mu}$ can be represented as

$$
L^{(+) i j \mu}=-\frac{1}{4} \epsilon_{m n}^{i j} \epsilon^{\mu \nu \rho \sigma} D_{\nu} H_{\rho \sigma}^{(+) m n},
$$

where $H^{(+) i j}{ }_{\mu \nu}$ is self-dual with respect to $(i j)$. From (2.14), we can obtain the following relation:

$$
L_{i j \mu}^{(+)}=2\left(e_{\mu[i} K_{j] k}^{(+)} k-K_{\mu[i j]}^{(+)}\right)
$$

with $K_{i j \mu}^{(+)}$being self-dual with respect to $(i j)$. Accordingly, the self-dual part of the scalar curvature is written as

$$
R^{(+)}=\frac{1}{2} R(e)+\frac{1}{2} L_{i j \mu}^{(+)} K^{(+) i j \mu}
$$

This expression of $R^{(+)}$should be compared to (2.13) for $R$.

We are now ready to separate the quadratic terms of torsion from the gravitational part of the chiral action of (2.2). Using (2.16) in (2.2), the chiral action can be rewritten as follows:

$$
S^{(+)}=\frac{1}{2} \int d^{4} x e\left[R(e)+L_{i j \mu}^{(+)} K^{(+) i j \mu}\right]+[\text { matter terms }]
$$

In the source-free case, the torsion identically vanishes and hence the chiral action of (2.17) is reduced to the Einstein-Hilbert action. If the matter terms exist, however, the imaginary part appear in the chiral action of (2.17).

We shall analyze the consistency of the field equations which are derived from a slightly generalized chiral action. Hayashi and Bregman proposed to extend the gravitational Lagrangian in Poincaré gauge theory by adding the most general kinetic Lagrangian $L_{T}$ of the tetrad field to the first-order Palatini Lagrangian:

$$
L_{G}=\frac{1}{2} R+L_{T}
$$

According to the relation $D_{[\mu} e_{\nu]}^{i}=K_{j[\mu}^{i} e_{\nu]}^{j}$, the $L_{T}$ is a bilinear form of the torsion (or equivalently the contorsion). Thus the most general expression of $L_{T}$ is given by

$$
L_{T}=\alpha L_{i j \mu} K^{i j \mu}+\beta v^{2}+\gamma a^{2}
$$


with $\alpha, \beta$ and $\gamma$ being three arbitrary parameters, when invariance under space inversion is required. 1 Here the vector $v_{i}$ and the axial vector $a_{i}$ are

$$
\begin{aligned}
& v_{i}=K_{i j}{ }^{j}=-\frac{1}{2} L_{i j}{ }^{j}, \\
& a_{i}=\frac{1}{3} \epsilon_{i j k l} K^{j k l}=-\frac{1}{6} \epsilon_{i j k l} L^{j k l},
\end{aligned}
$$

respectively, with $K_{i j k}=e_{k}^{\mu} K_{i j \mu}$ and $L_{i j k}=e_{k}^{\mu} L_{i j \mu}$. The gravitational Lagrangian of (2.18) can be rewritten as

$$
L_{G}=\frac{1}{2} R(e)+\left[\left(\alpha+\frac{1}{4}\right) L_{i j \mu} K^{i j \mu}+\beta v^{2}+\gamma a^{2}\right]
$$

by means of (2.13) and (2.19).

The chiral version of this generalization of the gravitational Lagrangian can be constructed in the following manner. The chiral kinetic Lagrangian of the tetrad field, $L_{T}^{(+)}$, is obtained by replacing the $L_{i j \mu}$ in (2.19) by $L_{i j \mu}^{(+)}$; namely,

$$
L_{T}^{(+)}=2\left(\alpha L_{i j \mu}^{(+)} K^{(+) i j \mu}+\beta v^{(+) 2}+\gamma a^{(+) 2}\right)
$$

where

$$
\begin{aligned}
& v_{i}^{(+)}=K_{i j}^{(+)}{ }_{i j}^{j}=-\frac{1}{2} L^{(+)}{ }_{i j}^{j}, \\
& a_{i}^{(+)}=\frac{1}{3} \epsilon_{i j k l} K^{(+) j k l}=-\frac{1}{6} \epsilon_{i j k l} L^{(+) j k l}
\end{aligned}
$$

with $K_{i j k}^{(+)}=e_{k}^{\mu} K_{i j \mu}^{(+)}$and $L_{i j k}^{(+)}=e_{k}^{\mu} L_{i j \mu}^{(+)}$. We note that since $K_{i j k}^{(+)}$is self-dual with respect to $(i j), a_{i}^{(+)}$and $v_{i}^{(+)}$are related each other as follows:

$$
a_{i}^{(+)}=\frac{2}{3} i v_{i}^{(+)}=\frac{1}{2}\left(a_{i}+\frac{2}{3} i v_{i}\right)
$$

The chiral action of (2.2) is now generalized to the following form:

$$
S^{(+)}=\int d^{4} x e L_{G}^{(+)}+[\text {matter terms }]
$$

$\dagger$ The $\alpha^{\prime}, \beta^{\prime}$ and $\gamma^{\prime}$ in eq.(4.2a) of Ref. [1] are related to the $\alpha, \beta$ and $\gamma$ of (2.19) by $\alpha^{\prime}=$ $(4 / 3) \alpha, \beta^{\prime}=\beta-(4 / 3) \alpha$ and $\gamma^{\prime}=\gamma+3 \alpha$. 
with the generalized chiral gravitational Lagrangian $L_{G}^{(+)}$being

$$
L_{G}^{(+)}=R^{(+)}+L_{T}^{(+)}
$$

With the help of (2.26) and (2.16), $L_{G}^{(+)}$can be rewritten as

$$
L_{G}^{(+)}=\frac{1}{2} R(e)+2\left[\left(\alpha+\frac{1}{4}\right) L_{i j \mu}^{(+)} K^{(+) i j \mu}+\left(\beta-\frac{4}{9} \gamma\right) v^{(+) 2}\right] .
$$

It should be noticed that this involves two parameters in contrast to the $L_{G}$ of $(2.22)$ with three parameters.

\section{Chiral Lagrangian of matter fields}

The Lagrangian of integer spin fields does not contain the Lorentz connection and its imaginary part vanishes as in the source-free case. Therefore, we restrict ourselves to half-integer spin fields, taking spin-1/2 and spin-3/2 fields as examples.

We start with two postulates for the matter coupling. Firstly, let us suppose that the matter Lagrangian is obtained by the minimal prescription; namely, by replacing ordinary derivatives acting on half-integer spin fields by covariant derivatives,

$$
\partial_{i} \rightarrow e_{i}^{\mu} D_{\mu}
$$

with

$$
D_{\mu}=\partial_{\mu}+\frac{i}{2} A_{i j \mu} S^{i j}
$$

and $S_{i j}$ standing for the $\mathrm{SL}(2, \mathbf{C})$ generator. 7

Secondly, we suppose that the chiral Lagrangian of half-integer spin fields is described by using the self-dual part of the Lorentz connection. According to the equation

$$
A_{i j \mu}^{(+)} S^{i j}=A_{i j \mu} S^{i j} \frac{1+\gamma_{5}}{2},
$$

\footnotetext{
${ }^{*}$ In our convention $S_{i j}=\frac{i}{4}\left[\gamma_{i}, \gamma_{j}\right]$ and $\left\{\gamma_{i}, \gamma_{j}\right\}=-2 \eta_{i j}$.
} 
this demands that only the terms expressed by $D_{\mu} \psi_{R}$ and/or $\bar{\psi}_{L} \overleftarrow{D}_{\mu}$ should appear in the matter Lagrangian. Here $\psi_{R}\left(\psi_{L}\right)$ is the right (left)-handed spinor field:

$$
\left\{\begin{array}{l}
\psi_{R}=\frac{1+\gamma_{5}}{2} \psi \\
\psi_{L}=\frac{1-\gamma_{5}}{2} \psi
\end{array}\right.
$$

We shall now see how the above postulates apply to the matter coupling. For example, let us consider a (Majorana) Rarita-Schwinger field $\psi_{\mu}$. Spin-1/2 fields can be treated in a similar manner.

The Lagrangian of a (Majorana) Rarita-Schwinger field in flat space is usually defined by

$$
L_{R S}^{(0)}=\frac{1}{2} \epsilon^{\mu \nu \rho \sigma} \bar{\psi}_{\mu} \gamma_{5} \gamma_{\rho} \partial_{\sigma} \psi_{\nu}
$$

If we apply the minimal prescription to (3.5), the expression of (3.5) becomes

$$
L_{R S}=\frac{1}{2} \epsilon^{\mu \nu \rho \sigma} \bar{\psi}_{\mu} \gamma_{5} \gamma_{\rho} D_{\sigma} \psi_{\nu}
$$

which is used in $N=1$ supergravity 10 - 12.

According to the second postulate, however, the Lagrangian (3.5) in flat space should be reexpressed only in terms of $\partial_{\mu} \psi_{R \nu}$ and/or $\bar{\psi}_{L \nu} \overleftarrow{\partial}_{\mu}$. In fact, the Lagrangian (3.5) can be rewritten as

$$
L_{R S}^{(0)}=-\epsilon^{\mu \nu \rho \sigma} \bar{\psi}_{R \mu} \gamma_{\rho} \partial_{\sigma} \psi_{R \nu}
$$

except for a total divergence term. Note that only the first derivative of $\psi_{R \nu}$ appears in (3.7), because $\psi_{\nu}$ is Majorana spinor. We can take (3.7) as the chiral Lagrangian in flat space, for which to apply the minimal prescription. Then the chiral Lagrangian of a (Majorana) Rarita-Schwinger field in curved space is given by

$$
L_{R S}^{(+)}=-\epsilon^{\mu \nu \rho \sigma} \bar{\psi}_{R \mu} \gamma_{\rho} D_{\sigma} \psi_{R \nu}
$$

In order to see the relation between the $L_{R S}$ of (3.6) and the $L_{R S}^{(+)}$of (3.8), we rewrite these Lagrangians by using the contorsion tensor and its self-dual part. Then 
the Lagrangians of (3.6) and (3.8) can respectively be reexpressed as

$$
\begin{aligned}
L_{R S} & =L_{R S}(e)+\frac{i}{4} \epsilon^{\mu \nu \rho \sigma} \bar{\psi}_{\mu} \gamma_{5} \gamma_{\rho} K_{i j \sigma} S^{i j} \psi_{\nu}, \\
L_{R S}^{(+)} & =L_{R S}(e)+\frac{i}{2} \epsilon^{\mu \nu \rho \sigma} \bar{\psi}_{\mu} \gamma_{5} \gamma_{\rho} K_{i j \sigma}^{(+)} S^{i j} \psi_{\nu},
\end{aligned}
$$

where $L_{R S}(e)$ is the Lagrangian of a (Majorana) Rarita-Schwinger field in general relativity. Comparing (3.10) with (3.9), we notice that the factor of the second term in (3.10) is twice that in (3.9), and the real part of (3.10) coincides with that of (3.9).

We can treat a Dirac field $\psi$ similarly. The Lagrangian of a Dirac field in curved space can be obtained by applying the minimal prescription to that in flat space. On the other hand, in conformity with the second postulate, the chiral Lagrangian of a Dirac field in flat space can be defined by the Lagrangian of a Dirac field which is rewritten only in terms of $\partial_{\mu} \psi_{R}$ and $\bar{\psi}_{L} \overleftarrow{\partial}_{\mu}$ except for a total divergence term. We also apply the minimal prescription to this chiral Lagrangian. Furthermore, in order to see the relation between the Lagrangian in curved space and the chiral one, we use the contorsion tensor and its self-dual part. The resulting Lagrangians, $L_{D}$ and $L_{D}^{(+)}$, are given by

$$
\begin{aligned}
L_{D} & =L_{D}(e)-\frac{1}{4} \bar{\psi}\left\{\gamma^{\mu}, S^{i j}\right\} K_{i j \mu} \psi, \\
L_{D}^{(+)} & =L_{D}(e)-\frac{1}{2} \bar{\psi}\left\{\gamma^{\mu}, S^{i j}\right\} K_{i j \mu}^{(+)} \psi,
\end{aligned}
$$

where $L_{D}(e)$ is the Lagrangian of the Dirac field in general relativity. It can be seen that the factor of the second term in (3.11) is twice that in (3.12), and the real part of (3.12) coincides with that of (3.11) as in the case of a (Majorana) Rarita-Schwinger field.

In the above two examples of a (Majorana) Rarita-Schwinger field and a Dirac field, the Lagrangian is linear in first derivatives of the field variables, and the torsion is coupled with these fields linearly. Thus let us suppose that the real Lagrangian of half-integer spin fields is written as

$$
L_{M}=L_{M}(e)+X_{i j \mu} K^{i j \mu}
$$

where $L_{M}(e)$ is the Lagrangian of half-integer spin fields in general relativity, and $X_{i j k}=X_{[i j] k}$ is a real tensor made of matter fields. Then, taking into account of the 
above comparison the real Lagrangian with the complex chiral one, we assume that the complex chiral Lagrangian of half-integer spin fields is expressed as follows:

$$
L_{M}^{(+)}=L_{M}(e)+2 X_{i j \mu} K^{(+) i j \mu}
$$

It is noted that only the self-dual part $X_{i j \mu}^{(+)}$contributes in (3.14).

\section{Solving with respect to the torsion}

The total chiral Lagrangian $L^{(+)}$is given by the sum of (2.29) and (3.14):

$$
\begin{aligned}
L^{(+)} & =L_{G}^{(+)}+L_{M}^{(+)} \\
& =\frac{1}{2} R(e)+L_{M}(e)+L_{\text {torsion }}^{(+)},
\end{aligned}
$$

where $L_{\text {torsion }}^{(+)}$is defined by

$$
L_{\text {torsion }}^{(+)}=2\left(\alpha^{\prime} L_{i j k}^{(+)} K^{(+) i j k}+\beta^{\prime} v^{(+) 2}+X_{i j k}^{(+)} K^{(+) i j k}\right)
$$

with $\alpha^{\prime}$ and $\beta^{\prime}$ being set as

$$
\alpha^{\prime}=\alpha+\frac{1}{4}, \quad \beta^{\prime}=\beta-\frac{4}{9} \gamma
$$

The imaginary part of the chiral Lagrangian $L^{(+)}$emerges only from the $L_{\text {torsion }}^{(+)}$ of (4.2). The Lagrangian of (4.2) can be divided into real and imaginary parts as follows:

$$
\begin{aligned}
L_{\text {torsion }}^{(+)}= & \alpha^{\prime} L_{i j k} K^{i j k}+\frac{1}{2} \beta^{\prime}\left(v^{2}-\frac{9}{4} a^{2}\right)+X_{i j k} K^{i j k} \\
& +i\left(-\frac{\alpha^{\prime}}{2} \epsilon_{i j m n} L_{k}^{i j k} K_{k}^{m n}-\frac{3}{2} \beta^{\prime} v_{i} a^{i}-\frac{1}{2} \epsilon_{i j m n} X_{k}^{i j k} K_{k}^{m n}\right) .
\end{aligned}
$$

On the other hand, the total real Lagrangian $L$, which is defined by the sum of (2.22) and (3.13), is

$$
L=L_{G}+L_{M}=\frac{1}{2} R(e)+L_{M}(e)+L_{\text {torsion }}
$$


with $L_{\text {torsion }}$ being defined by

$$
L_{\text {torsion }}=\alpha^{\prime} L_{i j k} K^{i j k}+\beta v^{2}+\gamma a^{2}+X_{i j k} K^{i j k}
$$

Comparing $L_{\text {torsion }}$ and $\operatorname{Re} L_{\text {torsion }}^{(+)}$of (4.4), we see that $\operatorname{Re} L^{(+)}$does not coincide with $L$ generally unless $\beta=-(4 / 9) \gamma$.

Now it is easy to solve the equation of the torsion. The results can be summarized as follows:

(1) If $K_{i j \mu}$ is taken as independent variable instead of $A_{i j \mu}$, it can be shown that the equation for $K_{i j \mu}$ derived from the $\operatorname{Re} L_{\text {torsion }}^{(+)}$of (4.4) is equivalent to that derived from $\operatorname{Im} L_{\text {torsion }}^{(+)}$of (4.4); namely, although $L^{(+)}$is complex, extra conditions do not appear for the equation for $K_{i j \mu}$.

(2) It can also be shown that the equation for $K_{i j \mu}^{(+)}$obtained from the $L_{\text {torsion }}^{(+)}$of (4.2) is compatible with the equation for $K_{i j \mu}$ derived from the $L_{\text {torsion }}^{(+)}$of (4.4).

(3) Varying the $L_{\text {torsion }}^{(+)}$of (4.2) with respect to $K_{i j \mu}^{(+)}$, we get the following equation:

$$
2 \alpha^{\prime} L_{i j k}^{(+)}+\frac{1}{2} \beta^{\prime}\left(\eta_{k[i} L^{(+)}{ }_{j] l}^{l}-\frac{i}{2} \epsilon_{i j k}^{l} L^{(+)}{ }_{l m}^{m}\right)=-X_{i j k}^{(+)}
$$

This equation can be solved with respect to $L_{i j \mu}^{(+)}$as

$$
L_{i j k}^{(+)}=-\frac{1}{2 \alpha^{\prime}}\left[X_{i j k}^{(+)}-\frac{f}{2}\left(\eta_{k[i} X^{(+)}{ }_{j] l}^{l}-\frac{i}{2} \epsilon_{i j k}{ }^{m} X^{(+)}{ }_{m l}^{l}\right)\right],
$$

where

$$
f:=\frac{\beta^{\prime}}{2 \alpha^{\prime}-\frac{3}{4} \beta^{\prime}} .
$$

From (4.8) one can derive $L_{i j \mu}$. Then the contorsion tensor $K_{i j \mu}$ can be obtained by using the relation of (2.12). Substituting these $L_{i j \mu}$ and $K_{i j \mu}$ back into (4.4), we see that $\operatorname{Re} L_{\text {torsion }}^{(+)}$and $\operatorname{Im} L_{\text {torsion }}^{(+)}$give four-fermion contact terms.

\section{Consistency of the field equations}


In order to get the explicit expression for $\operatorname{Im} L^{(+)}$, it is more convenient to write $\operatorname{Im} L^{(+)}$in terms of the torsion tensor. Using $X_{i j k}$, which can be read from (4.7), in the $\operatorname{Im} L_{\text {torsion }}^{(+)}$of $(4.4)$ gives

$$
\operatorname{Im} L^{(+)}=\frac{\alpha^{\prime}}{2} \epsilon_{i j m n} L^{i j k} K_{k}^{m n}+\frac{3}{2} \beta^{\prime} v_{i} a^{i}
$$

After a little calculation, (5.1) becomes

$$
\operatorname{Im} L^{(+)}=\frac{\alpha^{\prime}}{2} \epsilon_{i j m n} T^{k i j} T_{k}^{m n}+\frac{3}{2} \beta^{\prime} v_{i} a^{i}
$$

Let us now consider the cases of spin- $1 / 2$ and spin- $3 / 2$ fields. In the case of spin-1/2 fields, the torsion tensor is totally anti-symmetric:

$$
T_{k i j}=-\frac{1}{8 \alpha^{\prime}}\left(1+\frac{3}{4} f\right) \epsilon_{i j k l} \bar{\psi} \gamma_{5} \gamma^{l} \psi
$$

Substituting (5.3) into (5.2), we see that $\operatorname{Im} L^{(+)}$vanishes for arbitrary values of $\alpha^{\prime}$ and $\beta^{\prime}$.

Next for $N$-(Majorana) Rarita-Schwinger fields $\psi_{\mu}^{I}$ where $I$ runs from 1 to $N$, the torsion tensor becomes

$$
T_{k i j}=-\frac{i}{8 \alpha^{\prime}} \sum_{I}\left[\bar{\psi}_{i}^{I} \gamma_{k} \psi_{j}^{I}-\frac{f}{2} \eta_{k[i} \bar{\psi}_{j]}^{I} \gamma^{m} \psi_{m}^{I}+\frac{f}{8} \epsilon_{i j k l} \epsilon^{l m n r} \bar{\psi}_{m}^{I} \gamma_{r} \psi_{n}^{I}\right]
$$

Inserting this into $(5.2)$ gives

$$
\operatorname{Im} L^{(+)}=-\frac{1}{128 \alpha^{\prime}} \sum_{I, J} \epsilon^{\mu \nu \rho \sigma}\left[\left(\bar{\psi}_{\mu}^{I} \gamma_{k} \psi_{\nu}^{I}\right)\left(\bar{\psi}_{\rho}^{J} \gamma^{k} \psi_{\sigma}^{J}\right)-f e_{\rho}^{j} e_{k}^{\lambda}\left(\bar{\psi}_{\mu}^{I} \gamma_{j} \psi_{\nu}^{I}\right)\left(\bar{\psi}_{\sigma}^{J} \gamma^{k} \psi_{\lambda}^{J}\right)\right]
$$

In this case $\operatorname{Im} L^{(+)}$does not vanish in general.

The gravitational and matter field equations are affected by this $\operatorname{Im} L^{(+)}$of (5.5). If the tetrad is real, the Einstein equation and the Rarita-Schwinger equation are obtained by taking variation of $\operatorname{Re} S^{(+)}$with respect to the tetrad and the (Majorana) Rarita-Schwinger fields. Furthermore, $\operatorname{Im} S^{(+)}$yields algebraic equations:

$$
\sum_{I, J} f \epsilon^{\mu \nu \rho \sigma}\left(\bar{\psi}_{\mu}^{I} \gamma_{j} \psi_{\nu}^{I}\right)\left[e_{\rho}^{k} e_{\lambda}^{j} e_{i}^{\tau}\left(\bar{\psi}_{\sigma}^{J} \gamma^{i} \psi_{\tau}^{J}\right)-e_{\rho}^{j}\left(\bar{\psi}_{\sigma}^{J} \gamma^{k} \psi_{\lambda}^{J}\right)\right]=0
$$


for the tetrad field $e_{k}^{\lambda}$, and

$$
\sum_{J(J \neq I)} \epsilon^{\mu \nu \rho \sigma}\left[\bar{\psi}_{\rho}^{J} \gamma^{k} \psi_{\sigma}^{J}-f e_{\rho}^{k} e_{j}^{\lambda}\left(\bar{\psi}_{\sigma}^{J} \gamma^{j} \psi_{\lambda}^{J}+2 \bar{\psi}_{\sigma}^{I} \gamma^{j} \psi_{\lambda}^{I}\right)\right] \gamma_{k} \psi_{\nu}^{I}=0
$$

for a (Majorana) Rarita-Schwinger field $\psi_{\mu}^{I}$. In the derivation of (5.7), we have used the identity

$$
\epsilon^{\mu \nu \rho \sigma}\left(\bar{\psi}_{\rho}^{I} \gamma^{k} \psi_{\sigma}^{I}\right) \gamma_{k} \psi_{\nu}^{I} \equiv 0
$$

which is valid because of the Fierz identity. 冈 When $f \neq 0$ (namely, $v^{(+) 2}$-term in (4.2) is non-vanishing), there is the difficulty of overdetermination for $\psi_{\mu}^{I}$ from (5.6) and (5.7). On the other hand, when $f=0$ (namely, $v^{(+) 2}$-term in (4.2) is vanishing), there is no such problem for $N=1$ because both (5.6) and (5.7) disappear. However, for $N \geq 2$, there is the possible difficulty of overdetermination arising from (5.7). It should be noticed that these results are also valid for (Dirac) Rarita-Schwinger fields, because a complex (Dirac) Rarita-Schwinger field is equivalent with the two real (Majorana) Rarita-Schwinger fields.

\section{Summary}

We studied the consistency of the field equations by using the generalized chiral Lagrangian $L^{(+)}$of (4.1). Let us summarize our results in order.

[1] The generalized gravitational Lagrangian $L_{G}$ involves three parameters $\alpha, \beta$ and $\gamma$ besides the Newton gravitational constant, while its chiral part $L_{G}^{(+)}$has two parameters $\alpha^{\prime}=\alpha+1 / 4$ and $\beta^{\prime}=\beta-(4 / 9) \gamma$. The $\operatorname{Re} L_{G}^{(+)}$does not coincide with the $L_{G}$ generally unless $\beta=-(4 / 9) \gamma$.

[2] The equation of motion for the contortion $K_{i j \mu}$ derived from $\operatorname{Re} L^{(+)}$is equivalent to that derived from $\operatorname{Im} L^{(+)}$; namely, although $L^{(+)}$is complex, extra conditions do not appear for the equation of motion for $K_{i j \mu}$.

[3] The equation of motion for $K_{i j \mu}^{(+)}$derived from $L^{(+)}$is compatible with the equation of motion for $K_{i j \mu}$ derived from $L^{(+)}$.

* See, for example, eq.(7) in p.365 of Ref. 112. 
[4] Substituting $L_{i j \mu}$ obtained from the equation of motion for $K_{i j \mu}^{(+)}$back into $L^{(+)}$, the torsion part of $L^{(+)}$gives the four-fermion contact terms, of which the imaginary part is given by $(5.2)$.

As examples, the cases of spin-1/2 fields and (Majorana) Rarita-Schwinger fields were discussed with the following results.

[5] For spin-1/2 fields, the imaginary part of the generalized chiral Lagrangian is always vanishing for arbitrary values of $\alpha^{\prime}$ and $\beta^{\prime}$. Therefore the consistency problem does not arise in the case of spin- $1 / 2$ fields.

[6] For $N$-(Majorana) Rarita-Schwinger fields, $\operatorname{Im} L^{(+)}$is non-vanishing generally.

(a) In the case of $\alpha=\beta=\gamma=0$ (i.e. $L_{G}=(1 / 2) R$ and $\left.L_{G}^{(+)}=R^{(+)}\right)$, the imaginary part of the chiral Lagrangian does not vanish except for $N=1$. Thus the consistency problem arises for $N \geq 2$.

(b) In the general case of arbitrary values of $\alpha, \beta$ and $\gamma$, the imaginary part of the generalized chiral Lagrangian does not vanish unless the two conditions $f=0$ (namely, $v^{(+) 2}$-term in (4.2) is vanishing) and $N=1$ are satisfied. If $f \neq 0$, therefore, the consistency problem arises. When $N \geq 2$, there is such problem even if $f=0$.

These results (a) and (b) are also valid for (Dirac) Rarita-Schwinger fields. 


\section{References}

[1] Ashtekar A 1986 Phys. Rev. Lett. 57 2244; 1987 Phys. Rev. D36 1587

[2] Jacobson T and Smolin L 1987 Phys. Lett. 196B 39; 1988 Class. Quantum Grav. 5583

[3] Ashtekar A 1988 New Perspectives in Canonical Gravity (Naples: Bibliopolis)

[4] Ashtekar A 1991 Lectures on Non-Perturbative Canonical Gravity (Singapore: World Scientific)

[5] Ashtekar A, Romano J D and Tate R S 1989 Phys. Rev. D40 2572

[6] Jacobson T 1988 Class. Quantum Grav. 5923

[7] Capovilla R, Dell J, Jacobson T and Mason L 1991 Class. Quantum Grav. 8 41

[8] Schlling T 1994 Bibliography of Publications related to Classical and Quantum Gravity in terms of the Ashtekar Variables Preprint gr-qc/9409031

[9] Hayashi K and Bregman A 1973 Ann. Phys. (N.Y.) 75562

[10] Freedman D Z, van Nieuwenhuizen P and Ferrara S 1976 Phys. Rev. D13 3214 Freedman D Z and van Nieuwenhuizen P 1976 Phys. Rev. D14 912

[11] Deser S and Zumino B 1976 Phys. Lett. 62B 335

[12] van Nieuwenhuizen P 1981 Phys. Rep. 68189 